\title{
Morning vs evening dosing of the cathepsin $K$ inhibitor ONO-5334: effects on bone resorption in postmenopausal women in a randomized, phase 1 trial
}

\author{
R. Eastell ${ }^{1,5}$ • D.-J. Dijk ${ }^{2}$ - M. Small ${ }^{3}$ - A. Greenwood ${ }^{2} \cdot$ J. Sharpe $^{3} \cdot$ H. Yamada $^{3}$ •

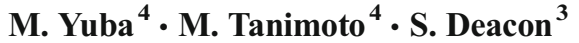

Received: 19 May 2015 / Accepted: 24 September 2015/Published online: 7 October 2015

(C) The Author(s) 2015. This article is published with open access at Springerlink.com

\begin{abstract}
Summary The cathepsin K inhibitor, ONO-5334, improves bone mineral density in postmenopausal women with osteoporosis. The effects of morning versus evening administration of ONO-5334 were investigated by measuring bone turnover marker levels in healthy postmenopausal women. Morning administration of ONO-5334 showed a more consistent suppressive effect on bone resorption than evening administration. Introduction Bone turnover is thought to be subject to circadian variation, and the efficacy of osteoporosis treatments may be optimized by regulating the time of dosing. This study assessed whether evening administration of the cathepsin $\mathrm{K}$ inhibitor, ONO-5334, had a differential effect on the bone turnover marker, C-terminal telopeptide of type I collagen (CTX-I), compared with morning administration.

Methods This was a single-center, single blind crossover study. Fourteen healthy postmenopausal women were assigned to receive ONO-5334 $150 \mathrm{mg}$ once daily for 5 days in each period; they were randomized to receive either evening doses in the first
\end{abstract}

Electronic supplementary material The online version of this article (doi:10.1007/s00198-015-3342-4) contains supplementary material, which is available to authorized users.

R. Eastell

r.eastell@sheffield.ac.uk

1 Academic Unit of Bone Metabolism, University of Sheffield, Sheffield, UK

2 Surrey Clinical Research Centre, University of Surrey, Guildford, UK

3 Ono Pharma UK Ltd, London, UK

4 Ono Pharmaceutical Co., Ltd, Osaka, Japan

5 Metabolic Bone Centre, Northern General Hospital, Herries Road, Sheffield, South Yorkshire S5 7AU, UK period and morning doses in the second or vice versa. Serum and urinary levels of CTX-I were measured throughout the study. Results Both regimens showed similar patterns of reduction in serum and urinary CTX-I; however, CTX-I suppression was more consistently $>60 \%$ over $24 \mathrm{~h}$ following morning administration. Morning administration led to $6 \%$ greater suppression of 24-h serum CTX-I area under the effect curve (AUE; 69 vs 63\%; $P<$ $.05)$ and $7 \%$ greater suppression of urinary CTX-I/creatinine AUE (93 vs $86 \% ; P<.01$ ) than evening administration. Higher plasma ONO-5334 concentrations were observed between 12 and $24 \mathrm{~h}$ postdose following morning administration, with mean trough concentrations for the morning and evening regimens at 9.4 and $4.0 \mathrm{ng} / \mathrm{mL}$, respectively. There were no safety findings of concern. Conclusion Morning dosing of ONO-5334 is more efficacious at reducing markers of bone turnover in healthy postmenopausal women than evening dosing.

Trial registration ClinicalTrials.gov: NCT01384188, registered on June 27, 2011

EudraCT: 2008-006284-37

Keywords Anti-resorptive $\cdot$ Bone resorption · Menopause · Osteoporosis

$\begin{array}{ll}\begin{array}{l}\text { Abbreviations } \\ \text { AUC }_{24 h} \text { Area under the concentration-time curve during } \\ \text { the dosing interval }\end{array} \\ \text { AUE } & \text { Area under the effect curve } \\ \text { BMD } & \text { Bone mineral density } \\ C_{\max } & \text { Maximum observed concentration } \\ \text { CTX-I } & \text { C-terminal telopeptide of type I collagen } \\ \text { IRT } & \text { Immediate-release tablet } \\ \text { SRT } & \text { Sustained-release tablet } \\ T_{\max } & \text { Time to maximum plasma concentration }\end{array}$




\section{Introduction}

Osteoporosis mainly arises in postmenopausal women and affects $>10$ million people in the USA; the incidence is set to increase as the population ages [1]. Osteoporosis is characterized by low bone mass disordered bone architecture, leading to an increased risk of vertebral and other fractures. Osteoporosis is associated with significant morbidity, and hip fractures as sequelae confer a 1-year mortality risk of 8.4-36\% primarily due to thromboembolic events [1].

An important contributory factor to low bone mass in osteoporosis is increased bone resorption. Antiresorptive drugs such as the bisphosphonates reduce bone resorption and are the current drug class of choice for the prevention of fractures in patients with osteoporosis [2]. However, bisphosphonates are not well tolerated by many patients, may cause abdominal discomfort or dyspepsia and are difficult to administer. Furthermore, chronic bisphosphonate therapy may enhance the risk of atypical femur fractures, although the overall incidence of fractures is reduced [3]. Hence, there is a need to develop novel, safe and efficacious treatments for osteoporosis.

Cathepsin $\mathrm{K}$ inhibitors including odanacatib [4] and ONO5334 are a new class of drugs against osteoporosis which are undergoing clinical development. ONO-5334 improved bone strength by preferentially increasing cortical bone mass in ovariectomized rats [5] and significantly improved cortical and trabecular bone mineral density (BMD) in postmenopausal women with osteoporosis following 24 months of therapy [6]. In healthy postmenopausal women, ONO-5334 $100 \mathrm{mg}$ sustained-release tablet (SRT) administered in the morning exhibited a median time to maximum plasma concentration $\left(T_{\max }\right)$ of $4 \mathrm{~h}$ and mean half-life of $15 \mathrm{~h}$ following repeat administration $[7,8]$, suggesting the feasibility of once-daily dosing.

Serum and urinary bone turnover markers such as $\mathrm{C}$ terminal telopeptide of type I collagen (CTX-I) peak in the early morning hours and reach nadir in the afternoon, demonstrating that bone resorption is highest at night in healthy and osteoporotic postmenopausal women [9-13].

Thus, evening administration of antiresorptive drugs such as ONO-5334, in contrast to the more conventional morning administration, is of interest because plasma drug levels may be higher at the time when bone resorption is greatest.

The influence of administration time of anti-osteoporosis drugs on efficacy varies. For instance, Blumsohn and coworkers [14] administered elemental calcium $1000 \mathrm{mg}$ at bedtime, which suppressed nighttime increases of bone resorption and reduced $24 \mathrm{~h}$ of excretion of bone resorption markers in premenopausal women, whereas morning administration had no effect. In addition, Karsdal and coworkers $[15,16]$ showed that oral calcitonin given between 17:00 and 22:00 exerted greater reductions of bone turnover markers compared with administration at 08:00. Odanacatib is administered once weekly, so the effects of administration time on efficacy would be of little value; however, ONO-5334 is intended as a oncedaily medication

This study investigated healthy postmenopausal women as a representative potential patient population with similar predominant features of gender, age and hormonal milieu of patients with osteoporosis, without the attendant concomitant treatments that might confound the results. The aim of this randomized, single blind, two-part crossover study was to investigate the effects of morning versus evening administration on the pharmacokinetics and pharmacodynamics of multiple doses of ONO-5334 150-mg SRT in healthy postmenopausal women.

\section{Methods}

\section{Study population}

Healthy female subjects aged 55-75 years with a body mass index of $19-30 \mathrm{~kg} / \mathrm{m}^{2}$ were enrolled. Subjects had been amenorrheic for $\geq 5$ years and had follicle-stimulating hormone $>30$ $\mathrm{IU} / \mathrm{L}$ and estrogen $<92 \mathrm{pmol} / \mathrm{L}$. Subjects had a sleep history (for $\geq 1$ month before screening) of regular bedtime and sleep duration, did not awake more than twice nightly for micturition, had no habit of daytime naps $>1 \mathrm{~h}$ and experienced no sleep complaint as determined by a Pittsburgh Sleep Quality Index (PSQI) [17] score of $>5$. Subjects were in good health at both screening visits between 41 and 10 days before the first dose.

To minimize possible confounding factors of exercise and sleep/wake (circadian) on bone turnover, exercise was restricted, and a regular sleep/wake cycle was scheduled. Subjects maintained bedtime at 22:30 and wake-up time at 06:30 throughout the study period. This applied at home from the second screening visit until admission to the clinical unit and during the entire 10-day residential period. A subjective sleep diary incorporating the Karolinska Sleepiness Scale [18] and actigraphy (as monitored by Actiwatch AW4; Cambridge Neurotechnology, Cambridge, UK) confirmed compliance to the sleep/wake schedule and limited exercise regimen.

Informed consent was obtained from all individual participants included in the study. The study was approved by a local review board (London Westminster Research Ethics Committee) and conducted in accordance with the Declaration of Helsinki and Good Clinical Practice guidelines.

\section{Study design}

This was a randomized, single blind, single center, two-period crossover study (protocol: ONO-5334POE010; ClinicalTrials.gov: NCT01384188) between June and December 2011 at the Surrey Clinical Research Centre, with recruitment and initial screening performed at another site. Subjects received a single dose of oral ONO-5334 150-mg 
SRT (Ono Pharmaceutical Co, Ltd, Osaka, Japan) once-daily for 5 days in each treatment period.

Subjects were randomly assigned to receive evening ONO5334 administration in the first period and morning administration in the second or vice versa. Randomization codes were provided by quintiles and generated by the computer software package Statistical Analytical System (SAS) version 9.2. Treatment periods were separated by a washout of $\geq 10$ days. To maintain blinding, evening dose subjects took a placebo in the morning and morning dose subjects took a placebo in the evening.

All meals were standardized throughout the treatment period with nutritionally matched morning and evening meals. Breakfast, lunch and dinner were served at 07:00, 13:00 and 19:00, respectively. All subjects were given the same meal at each sitting and encouraged to finish the meal. Study agents were administered $30 \mathrm{~min}$ after the subjects finished morning or evening meals, at 08:00 and 20:00, respectively. A final follow-up visit was scheduled 5-8 days after the last dose.

\section{Pharmacokinetic sample collection and analysis}

Blood samples for measurement of plasma ONO-5334 concentration were collected via intravenous cannula over $12 \mathrm{~h}$ on day 1 and over $24 \mathrm{~h}$ on day 5 . Samples were taken once hourly between 1 and $6 \mathrm{~h}$ postdose. Sample time-points were relative to the ONO-5334 active dose (either morning or evening) in each treatment period. To minimize overnight disturbance, long-line extension tubing was attached to the cannula, allowing sample collection in another room.

ONO-5334 was extracted from plasma samples using a solid-phase extraction column followed by quantification. Plasma concentrations of ONO-5334 were determined by a validated liquid chromatography-tandem mass spectroscopy method with a lower limit of quantification at $0.02 \mathrm{ng} / \mathrm{mL}$. The assay was performed by Sumika Chemical Analysis Service (Osaka, Japan).

Pharmacokinetic parameters were estimated using noncompartmental methods (Phoenix WinNonlin version 6.1; Pharsight, Mountain View, CA, USA) and included maximum observed concentration $\left(C_{\max }\right), T_{\max }$ and area under the concentration-time curve during the dosing interval $\left(\mathrm{AUC}_{24 \mathrm{~h}}\right)$.

A scatter plot of individual plasma ONO-5334 concentrations versus serum CTX-I values (\% change from baseline, defined as day -1) was produced, and a squared correlation coefficient $(R 2)$ value was calculated. All data retrieved on day 5 in both treatment periods were included.

\section{Pharmacodynamic sample collection and analysis}

Blood and urine samples were collected for analysis of biochemical markers of bone turnover (Serum and Urine
CrossLaps ELISA; Immunodiagnostic Systems, Boldon, UK). Baseline serum samples for CTX-I were taken via an intravenous cannula on day -1 for the morning dosing period at $08: 00,10: 30,14: 00,16: 00,18: 00,20: 00,22: 30,02: 00$, 04:00, 06:00 and then again at 08:00 just before dosing on day 1 . For the evening dosing period, sampling started at 20:00 on day -1 and then at similar intervals until 20:00 on day 1 just before dosing. Baseline urine samples were collected on day -1 for the morning dosing period from 08:00 $10: 30,10: 30-14: 00,14: 00-16: 00,16: 00-18: 00,18: 00$ $20: 00,20: 00-22: 30,22: 30-04: 00$ to $04: 00-08: 00$. For the evening dosing period, sampling started at 20:00 and then at similar collection intervals until 20:00 on day 1 just before dosing.

Serum and urine samples were taken postdose on days 1 and 5 at 08:00 for the morning dosing and 20:00 for the evening dosing. Blood samples for analysis of serum CTX-I were taken at $2.5,6,8,10,12,14.5,18,20,22$ and $24 \mathrm{~h}$ postdose on days 1 and 5. Urine was collected for analysis of urinary CTXI over $0-2.5,2.5-6,6-8,8-10,10-12,12-14.5,14.5-20$ and 20-24 h postdose on days 1 and 5 . If subjects had not voided urine between 22:30 and 04:00 on overnight collection days, they were woken just before 04:00 to provide a urine sample. A dim red light $(<10 \mathrm{~lx})$ minimized impact on circadian status during scheduled lights-off periods.

Pharmacodynamic analyses were conducted by Charles River Laboratories (Edinburgh, UK). Parameters included derived total 24-h urinary CTX-I (area under the effect curve; AUE) and derived total 24-h serum CTX-I (AUE). Urinary CTX-I measurements were adjusted for urinary creatinine.

\section{Safety assessments}

Clinical laboratory tests, vital signs, 12-lead electrocardiograms and physical examinations were performed in each treatment period on admission and at follow-up; vital signs were measured at baseline, on all treatment days and upon discharge. All adverse events were coded using Medical Dictionary for Regulatory Activities version 14.1.

\section{Statistical analysis}

Pharmacodynamic and safety data were analyzed using SAS version 9.2. Urinary and serum CTX-I data were summarized using descriptive statistics. AUEs for urinary and serum CTXI were compared for morning versus evening regimens by ANCOVA. In a post-hoc analysis, urinary and serum CTX-I $\%$ change from clock time-matched baseline data were compared for morning versus evening regimens using repeatedmeasures approach.

Pharmacokinetic parameters were analyzed using SAS version 9.1.3 and 9.2. $C_{\max }, T_{\max }$ and $\mathrm{AUC}_{24 \mathrm{~h}}$ were compared between morning and evening administration on day 5 . 
Geometric mean ratios (morning/evening) and 90\% CIs were calculated using ANOVA. $T_{\max }$ was analyzed by Wilcoxon signed rank test at a two-sided significance level of $5 \%$. Safety data were not formally analyzed. Power calculations are difficult to apply to circadian studies and were not performed for this study. The sample size was chosen based on the results of Blumsohn and colleagues [14].

\section{Results}

\section{Pharmacokinetics}

Eleven subjects completed the study (Table 1) and were included on the pharmacokinetic and pharmacodynamics analyses. Maximum plasma ONO-5334 levels were observed later following evening administration than with the morning regimen (Fig. 1). Mean plasma ONO-5334 concentrations were lower between the 12- and 24-h postdose time-points on day 5 following evening administration compared with morning administration, with mean trough concentrations of approximately 4.0 and $9.4 \mathrm{ng} / \mathrm{mL}$, respectively. On day 5 following morning and evening dosing, $T_{\max }$ was observed at 2.0 and $5.0 \mathrm{~h}$ postdose, respectively (Table 2 ). A significant difference in $T_{\max }$ was observed between morning and evening dosing $(P=.002)$, although there was no significant difference in $C_{\max }$ and $\mathrm{AUC}_{24 \mathrm{~h}}$.

Table 1 Baseline demographics: safety population

\begin{tabular}{lc}
\hline & All subjects $(N=14)$ \\
\hline Enrolled, $n$ & 14 \\
Completed, $n(\%)$ & $11(79)$ \\
Age (years), mean [SD] & $65.0(7.7)$ \\
Height $(\mathrm{m})$, mean $[\mathrm{SD}]$ & $1.63(0.06)$ \\
Weight $(\mathrm{kg})$, mean $[\mathrm{SD}]$ & $64.6(7.1)$ \\
Body mass index $\left(\mathrm{kg} / \mathrm{m}^{2}\right)$, mean $[\mathrm{SD}]$ & $24.5(2.8)$ \\
Serum CTX-I $\left.(\mu \mathrm{g} / \mathrm{L}), \mathrm{mean}^{\mathrm{SSD}}\right]^{\mathrm{a}}$ & \\
08:00 & $0.50(0.17)$ \\
20:00 & $0.35(0.09)$ \\
Total 24 h (AUE) & $10.7(2.9)$ \\
Urine CTX-I/Cr $(\mu \mathrm{g} / \mathrm{mmol} \mathrm{Cr*h})$, mean $[\mathrm{SD}]^{\mathrm{a}}$ & \\
08:00-10:30 & $70.5(25.6)$ \\
20:00-22:30 & $69.3(31.7)$ \\
Total 24 h (AUE) $(\mu \mathrm{g} / \mathrm{mmol} \mathrm{Cr})$ & $1651.9(671.3)$ \\
\hline
\end{tabular}

All subjects were Caucasian women

$S D$ standard deviation, $C T X-I$ C-terminal telopeptide of type I collagen, $A U E$ area under the pharmacodynamic effects time curve, $\mathrm{Cr}$ creatinine

${ }^{a}$ Serum and urinary CTX-I were measured on the day before the first dose (day -1 , period 1 and period 2)

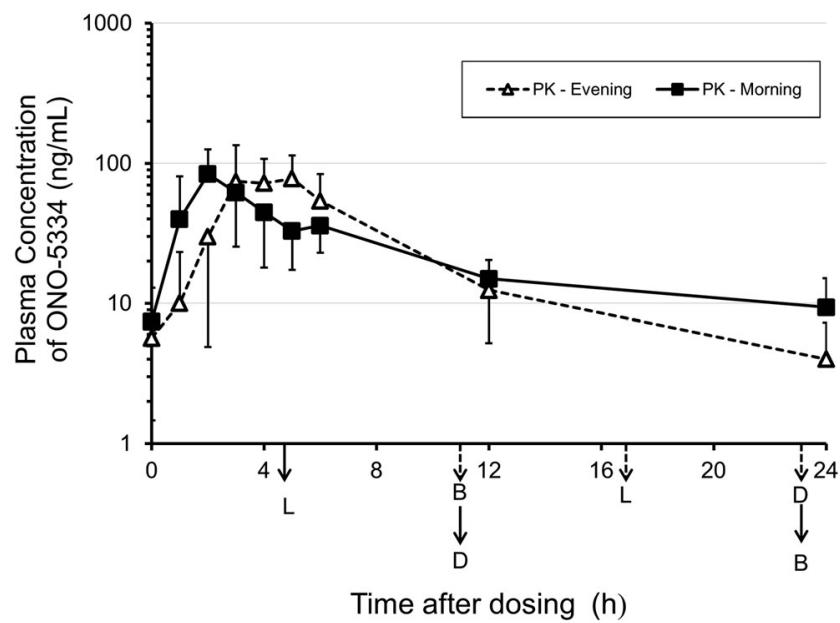

Fig. 1 Mean plasma concentration of ONO-5334 on day 5 (per protocol set). Values are expressed as mean (SD). Mealtimes are indicated with solid and dashed arrows for the morning and evening dosing groups, respectively. $P K$ pharmacokinetics, $B$ breakfast, $L$ lunch, $D$ dinner

\section{Pharmacodynamics}

On day 5 , there was a marked difference between morning versus evening dosing in serum CTX-I profile across $24 \mathrm{~h}$ (time-matched against each individual's own day -1, 24-h baseline profile; Fig. 2a). Although evening administration initially showed a larger suppressive effect on bone resorption, this was not sustained over $24 \mathrm{~h}$. Morning administration showed consistent suppression on serum CTX-I across $24 \mathrm{~h}$ compared with evening administration. Repeated-measures ANCOVA of the \% change from baseline for serum CTX-I on day 5 showed a greater reduction with morning compared with evening administration $(P=.004)$.

The morning dosing regimen showed a transient increase in serum CTX-I at $8 \mathrm{~h}$ postdose on day 5 , with levels decreasing thereafter. Interestingly, a similar slight peak in serum CTX-I was observed at $20 \mathrm{~h}$ after the evening dose, both of which corresponded to $3 \mathrm{~h}$ after lunch was served.

There was a marked difference in effect between morning and evening administration on the day 5 urinary CTX-I profile adjusted for urinary creatinine across $24 \mathrm{~h}$ (time-matched against each individual's own day $-1,24-\mathrm{h}$ baseline values; Fig. 2b). There was a more consistent suppressive effect on urinary CTX-I across $24 \mathrm{~h}$ following morning compared with evening administration, with less suppression observed at $12 \mathrm{~h}$ postdose and thereafter with the latter regimen. Repeatedmeasures ANCOVA of the \% change from baseline for urinary CTX-I/creatinine on day 5 showed a significantly greater reduction on morning compared with evening administration $(P=.0002)$.

Summarizing the data over $24 \mathrm{~h}$ using AUE analyses, differences between morning and evening dosing appeared of a lesser magnitude, although they retained statistical significance (Table 3). Morning administration achieved significantly greater 
Table 2 Pharmacokinetics of ONO-5334, day $5(n=11)$

\begin{tabular}{llccc}
\hline Dose $(\mathrm{mg})$ & Administration & $\begin{array}{l}C_{\max }(\mathrm{ng} / \mathrm{mL}) \\
\text { Mean (SD) }\end{array}$ & $\begin{array}{l}T_{\max }(\mathrm{h}) \\
\text { Median [range] }\end{array}$ & $\begin{array}{l}\mathrm{AUC}_{24 \mathrm{~h}}(\mathrm{ng} \mathrm{h} / \mathrm{mL}) \\
\text { Mean (SD) }\end{array}$ \\
\hline 150 & Morning & $99.5(45.3)$ & $2.0[1.0-3.0]$ & $582(196)$ \\
150 & Evening & $107.0(52.0)$ & $5.0[2.0-6.0]$ & $591(204)$ \\
$\mathrm{M} / \mathrm{E}^{\mathrm{a}}(90 \% \mathrm{CI})^{\mathrm{b}}$ & & $0.91(0.78-1.05)$ & $P=.0020^{\mathrm{c}}$ & $0.99(0.88-1.11)$ \\
\hline
\end{tabular}

$C_{\max }$ maximum observed concentration, $T_{\max }$ time to reach $C_{\max }, A U C_{24 h}$ area under the concentration-time curve during the dosing interval, $S D$ standard deviation, $M / E$ morning dosing/evening dosing, $C I$ confidence interval

${ }^{\mathrm{a}}$ Geometric mean ratio

${ }^{\mathrm{b}} 90 \%$ confidence interval of the geometric mean ratio

${ }^{\mathrm{c}}$ Wilcoxon signed rank test

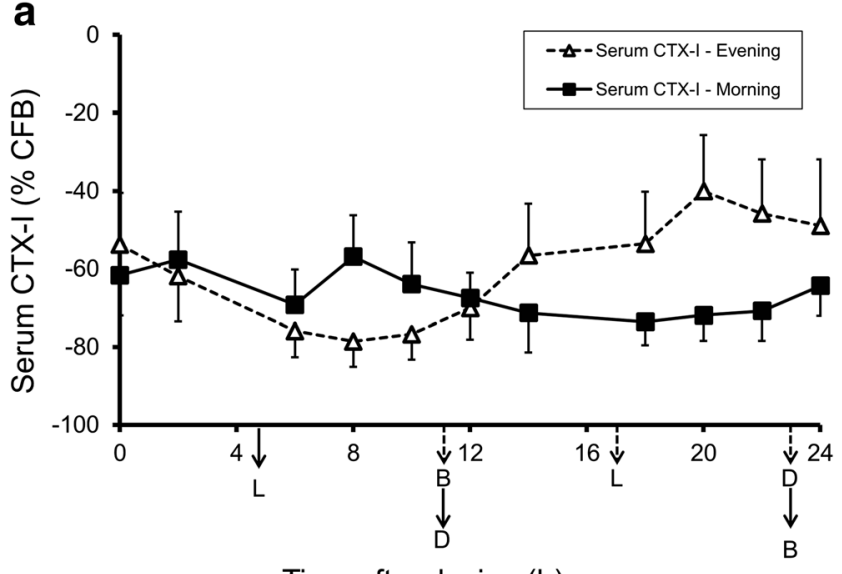

Time after dosing $(\mathrm{h})$

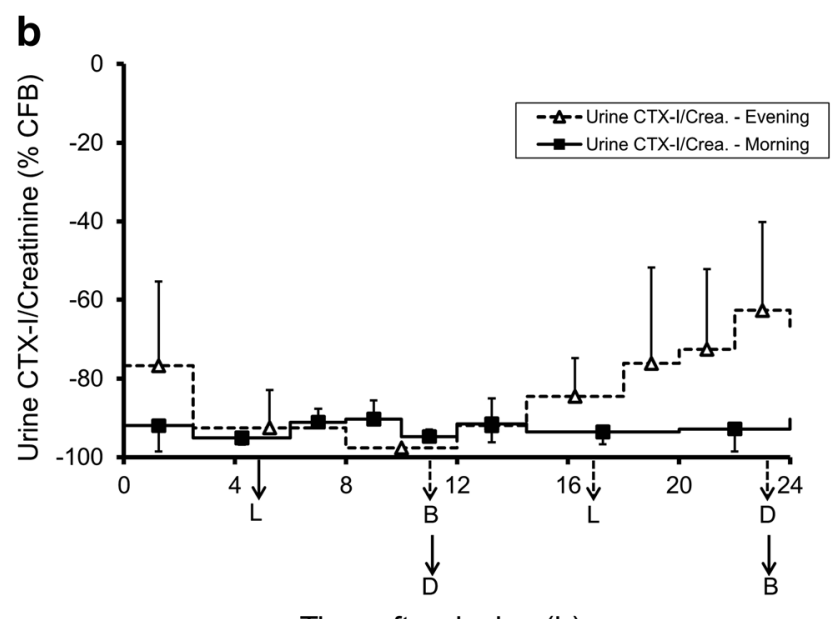

Time after dosing $(\mathrm{h})$

Fig. 2 a Serum CTX-I (time-matched \% change from baseline) after 5 days of ONO-5334 administration. Values are expressed as mean (SD). Mealtimes are indicated with solid and dashed arrows for the morning and evening dosing groups, respectively. $C T X-I \mathrm{C}$-terminal telopeptide of type I collagen, $C F B$ change from baseline, $B$ breakfast, $L$ lunch, $D$ dinner. $N=11$. b Urinary CTX-I/creatinine (time-matched \%CFB) after 5 days of ONO-5334 administration. Values are expressed as mean (SD). Mealtimes are indicated with solid and dashed arrows for the morning and evening dosing groups, respectively. $C T X-I$ C-terminal telopeptide of type I collagen, $C F B$ change from baseline, $B$ breakfast, $L$ lunch, $D$ dinner. $\mathrm{N}=11$ suppression of derived total 24-h serum CTX-I (AUE) than evening administration $(P=.020)$. For urine-derived total 24-h CTX-I/creatinine (AUE), morning administration also showed greater reduction than evening administration $(P=.009)$.

Serum CTX-I was suppressed after morning and evening administration of ONO-5334, with lowest CTX-I levels observed at $8 \mathrm{~h}$ postdose. Furthermore, after $24 \mathrm{~h}$ of dosing, CTX-I suppression was greater in the morning group. Figure 3 displays time-resolved CTX-I measurements after morning (Fig. 3a) and evening (Fig. 3b) administration of ONO-5334.

Figure 4 displays a scatter plot showing the correlation between plasma ONO-5334 levels and extent of CTX-I suppression on day 5. Data indicate that a plasma ONO-5334 concentration $\geq 10 \mathrm{ng} / \mathrm{mL}$ is required to produce $60 \%$ reduction of serum CTX-I.

\section{Study population and safety}

Fourteen female Caucasian subjects (mean age 65 years) were enrolled, and 11 completed the study (Table 1). Three subjects withdrew prematurely, two because of adverse events and one withdrew consent after 5 days of evening administration. Two of the three subjects withdrawn from the study were in the morning group, and the remaining subject was in the evening group. All three subjects had completed dosing in the first period but were withdrawn before the second dosing period (Supplementary Table 1). Fourteen subjects were included in the safety analysis.

Fourteen subjects reported 79 adverse events. The most frequent adverse event was procedural site reaction associated with the intravenous cannula (eight subjects, 57\%; Supplementary Table 1). All adverse events resolved. Procedural site reaction was more frequently reported after evening administration (seven subjects [54\%] compared with four subjects [33\%] after morning administration). Nausea was more frequent after morning administration (four subjects [33\%] compared with one subject [8\%] after evening 
Table 3 Serum and urinary CTX-I percent change from baseline on day 5 using AUE analysis over $24 \mathrm{~h}$

\begin{tabular}{lccr}
\hline Parameter & Morning (LS Mean) & Evening (LS Mean) & Morning vs Evening \\
\hline Serum CTX-I: AUE & -68.8 & -62.9 & -5.9 \\
Urine 24 h CTX-I/Cr: AUE & -92.6 & -85.8 & -6.8 \\
\hline
\end{tabular}

$N=11$. Analysis of covariance model including regimen, cohort, period and dosing sequence as fixed effects, baseline variable (day -1$)$ as a covariate and subject within sequence as a random effect

$C T X-I$ C-terminal telopeptide of type I collagen, $L S$ least squares, $C I$ confidence interval, $C r$ creatinine, $A U E$ area under the pharmacodynamic effects time curve

administration). Of adverse events that were considered as possibly treatment-related, nausea was most frequently reported (three subjects [25\%] following morning administration; one subject [8\%] following evening administration).

During the study, only one subject required concomitant medication of E45 cream. This was for a rash on both arms associated with cannula dressings. Two subjects were withdrawn from treatment as a result of mild adverse events (rash and aquagenic urticaria), considered as possibly related to
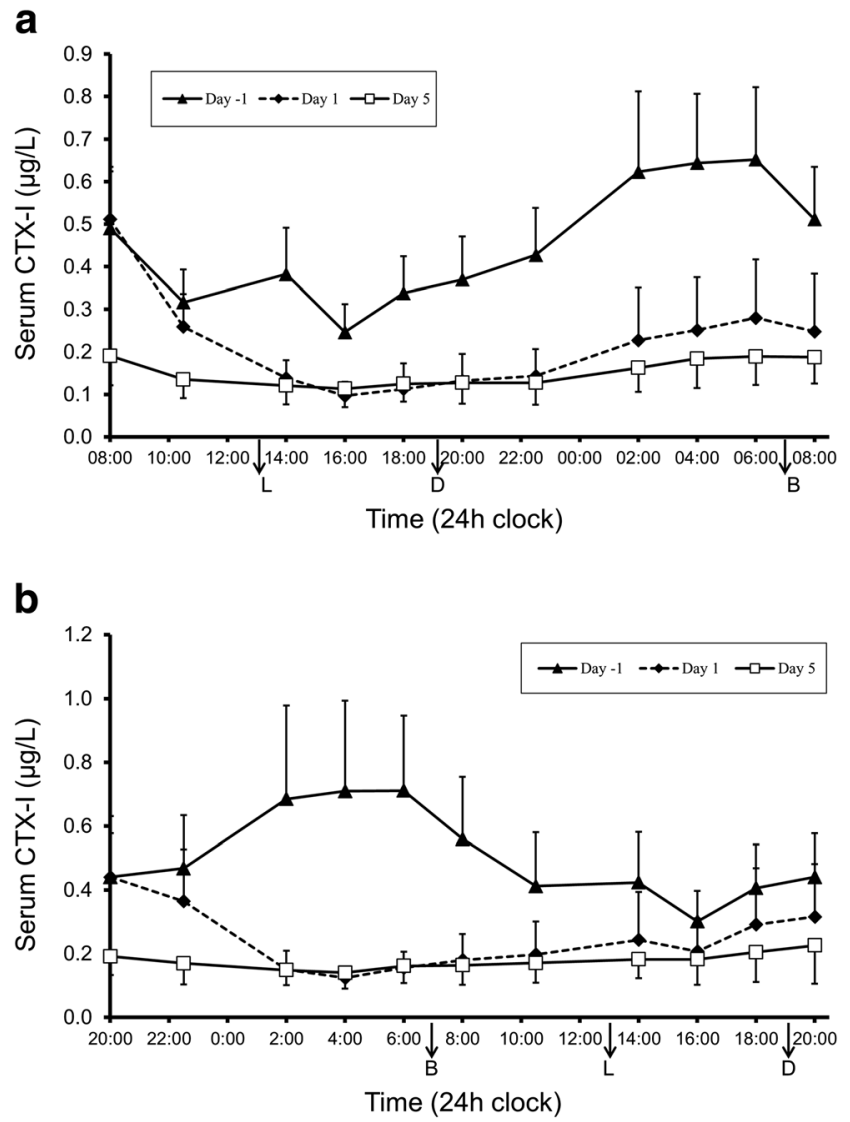

Fig. 3 a Time course of serum CTX-I levels after morning administration of ONO-5334 at 8:00 hours, $N=12$. b Time course of serum CTX-I levels after evening administration of ONO-5334 at 20:00 hours, $N=13$. Filled triangle indicates predose CTX-I levels (day 1), filled diamond indicates CTX-I levels on the day of first dosing (day 1), and open square indicates CTX-I levels on day 5. Mealtimes are indicated with arrows. $C T X-I \mathrm{C}$-terminal telopeptide of type I collagen, $B$ breakfast, $L$ lunch, $D$ dinner
ONO-5334 treatment. The rash on the eyelids was reported following 5 days of morning administration and resolved without further treatment after 11 days. Following investigation, this subject had a positive anti-nuclear antibody test, suggesting a possible autoimmune disorder. The case of aquagenic urticaria occurred 2 days after completion of 5 days' evening drug administration and resolved without further treatment after $1.5 \mathrm{~h}$. There were no clinically relevant laboratory safety data, vital signs, electrocardiogram or physical examinations.

\section{Discussion}

This study explored whether 5 days' administration of the novel cathepsin K inhibitor ONO-5334 150-mg SRT in the evening, when osteoclast activity begins to rise, resulted in a differential effect on pharmacodynamic markers of bone resorption compared with morning administration. Morning administration of ONO-5334 resulted in greater suppression of bone resorption markers over a $24-\mathrm{h}$ period than the same

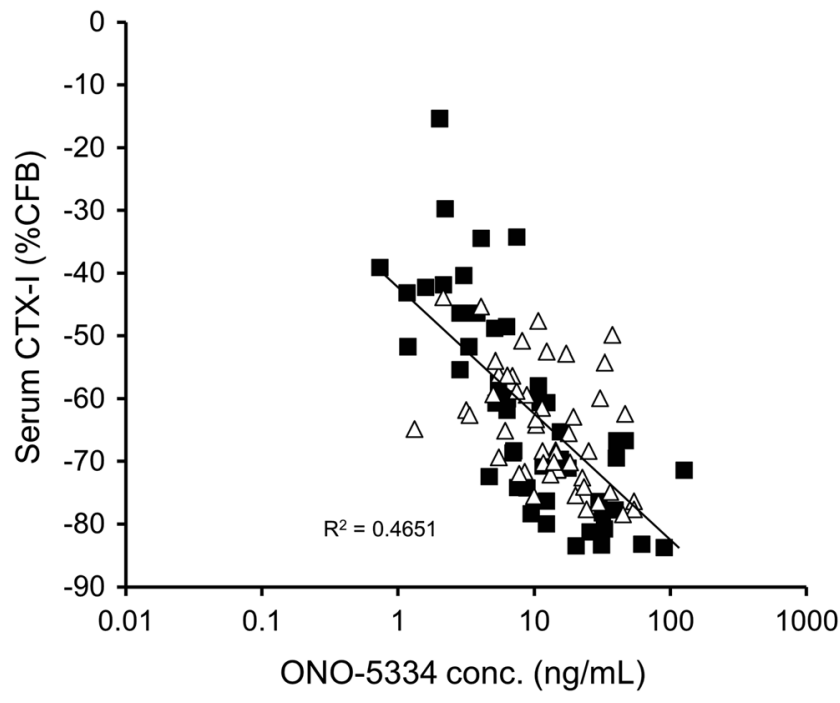

Fig. 4 Scatter plot of plasma ONO-5334 concentration versus serum CTX-I (\%CFB) on day 5. Open triangle morning dosing, filled square evening dosing. $C T X-I \mathrm{C}$-terminal telopeptide of type I collagen, $C F B$ change from baseline 
treatment given in the evening. These findings relate to the fed state and with ONO-5334 150-mg administered in sustainedrelease tablet formulation.

Markers of bone turnover such as serum CTX show distinct circadian patterns $[12,13]$. In women with osteoporosis, nocturnal serum calcium levels are sustained by increased bone resorption, in contrast to healthy postmenopausal women who display decreased serum calcium and lower urinary calcium excretion during nighttime [9]. Evening administration of antiresorptive drugs might enhance their efficacy because drug levels may be higher when resorption is greatest. The primary objective of the present study was to investigate the effects of ONO-5334 morning versus evening administration on pharmacodynamic parameters including serum and urinary CTX-I.

Although evening administration of ONO-5334 for 5 days initially had a greater suppressive effect on serum CTX-I than morning administration, this was not protracted and began to diminish $12 \mathrm{~h}$ postdose (evening administration). The reduction of serum CTX-I after morning administration was more consistent across all time-points. The urinary CTX-I profile showed more consistent suppression across all collection time-points following morning administration of ONO-5334. Following evening administration, suppression of urinary CTX-I was less apparent in urine collected $\geq 14 \mathrm{~h}$ postdose. Post-hoc analysis demonstrated that morning administration had significantly greater suppression of serum CTX-I and urinary CTX-I/creatinine than evening administration $(P<.01$ and $P<.001$, respectively). The effect of oral calcitonin administered in the morning, pre-dinner and in the evening on serum CTX-I in healthy postmenopausal women was reported by Karsdal et al. [15]. This showed a reduction in CTX-I, but the results differed in two ways from the present study. The first difference was that pre-dinner and evening doses of calcitonin resulted in greater CTX-I suppression than morning dosing. Secondly, the impact on bone resorption after oral calcitonin lasted for about $12 \mathrm{~h}$, in contrast to $24 \mathrm{~h}$ (or more) for ONO-5334.

The difference between morning and evening administration was less marked in AUE analyses across the 24-h period, although significant differences were observed. Morning administration led to $6 \%$ greater suppression of derived total 24-h serum CTX-I AUE and 7\% greater suppression of derived total 24-h urinary CTX-I/creatinine AUE than evening administration. Variation between samples was low; however, evening CTX-I levels showed highest variation, which has been observed in previous studies [19]. Standard deviations for the CTX-I assay employed in this study were comparable to references of intra-individual variation [20,21]. We considered that there may be an interaction between the effect of feeding and the effect of the drug, but we did not find the large decreases in CTX that are usually reported after meals
(Fig. 2a). This is likely due to the large suppression of CTXI by the drug.

Reductions from baseline in derived total 24-h serum CTXI AUE were consistent with previous reports on cathepsin $\mathrm{K}$ inhibitors, balicatib and odanacatib. In postmenopausal women, serum CTX was reduced by $61 \%$ in a 1 -year study on balicatib [22] and by $62-66 \%$ following single or repeated doses of odanacatib for 3 weeks $[23,24]$. In postmenopausal women with low BMD, once-weekly odanacatib elicited $>60 \%$ reductions of serum CTX-I for most of the first year of treatment [25] and continuous 55\% reductions were reported following 5 years of treatment [4]. In the OCEAN Study, bisphonate alendronate and ONO-5334 both exerted similar suppressive effects on bone resorption markers in women with osteoporosis $[26,27]$. Serum CTX-I decreases of $67-70 \%$ were reported following 30-36 months of alendronate therapy $[28,29]$, and urinary CTX-I was reduced by $53 \%$ following 12 weeks of alendronate treatment in healthy postmenopausal women [30].

Reduction in fracture rate is the goal for prevention and treatment of osteoporosis. An increase in BMD and suppression of bone resorption markers may achieve this. CTX-I is the reference marker of bone resorption used to evaluate experimental osteoporosis treatments [31]. Odanacatib has shown similar suppression of serum CTX-I to that observed with ONO-5334 (approximately 60\%), and both show clear increases in BMD [24, 25]. Other antiresorptive agents show similar magnitudes of serum CTX-I suppression of approximately $60-70 \%$ [32-34]. From a clinical standpoint, $60 \%$ suppression of serum CTX-I for as long as possible following ONO-5334 administration seems beneficial. ONO-5334 300mg IRT increased BMD in women with osteoporosis [26, 27] and has shown $>60 \%$ reduction from baseline in serum CTX-I in healthy postmenopausal women [35]. Therefore, the difference in the proportion of the day when the suppressive effect of ONO-5334 on serum CTX-I exceeded approximately $60 \%$ in the present study (that is, across the entire 24-h period following morning administration versus around half that after evening dosing) may be clinically important.

Differences in pharmacodynamic effects between the regimens in the present study were paralleled by differences in pharmacokinetic profiles. Higher plasma ONO-5334 concentrations were observed between 12 - and 24 -h postdose following 5 days of morning administration. This sustained plasma concentration may explain the increased serum and urinary CTX-I suppression observed toward the end of the 24-h period. The significantly $(P=.002)$ later $T_{\max }$ observed on day 5 after evening compared with morning dosing was considered attributable to delayed gastric emptying in the evening compared with morning [36, 37]. The disappearance rate of drug appeared to be more rapid following evening administration; we speculate that there may be diurnal changes in the clearance rate of ONO-5334. 
In the present study, we observed a correlation between plasma ONO-5334 levels and the extent of CTX-I suppression; our data indicate that a plasma ONO-5334 concentration $\geq 10 \mathrm{ng} / \mathrm{mL}$ is required to produce $>60 \%$ reduction of serum CTX-I at any given time-point.

Subjects randomized to morning administration in the first period had significantly higher serum CTX-I before dosing in the second period (following washout period) compared with subjects randomized to the evening regimen in the first period. This carryover effect may be attributed to greater suppression during the first period, followed by a rebound effect where CTX-I levels increased to above baseline after dosing was stopped. Odanacatib and denosumab also show rebound effects $[27,38,39]$. To account for baseline differences, morning versus evening comparisons were performed with $(P<0.05)$ and without $(P<0.01)$ adjustment for baseline, and the results were similar. It should also be noted that baseline bone turnover has been demonstrated to have no effect on the efficacy of ONO-5334 [26].

Treatment-related adverse events were more frequent after morning administration - particularly nausea, which occurred in three of 12 subjects and one of 13 subjects following morning and evening administration. However, the sample size was small, and the study was not powered to compare adverse event frequency. Following 12 months of evening dosing in 285 subjects in the OCEAN Study, the adverse event profile of ONO-5334, including nausea, was similar to placebo [26]. Procedural site reactions observed in eight subjects were related to insertion of the intravenous cannula and not to ONO5334 treatment per se. Two subjects were withdrawn from treatment as a result of mild rash and aquagenic urticaria that were considered as possibly related to ONO-5334; these did not resemble the morphea reported with balicatib [40]. The safety profile of ONO-5334 as regards to derangements of connective tissue will be closely monitored in future studies.

One limitation of the study concerned blinding; the more intensive blood and urinary sampling may have allowed subjects to suspect whether they had received active study medication in the morning or the evening. However, subjects were not explicitly informed what treatments they were receiving at the time of dosing; therefore, the study may be considered single blind.

Women with osteoporosis have decreased bone density and higher CTX-I levels than otherwise healthy women [41]. We have previously shown that baseline CTX-I levels are not predictive of how patients with osteoporosis respond to antiresorptive therapy with ONO-5334 [23]. In the same study, we found no association between baseline BMD (in the spine or hip) on ONO-5334 plasma levels or suppression of bone resorption by ONO-5334 (data unpublished). This suggests that the results of the current study on healthy postmenopausal women may also be applied to postmenopausal osteoporotic patients. However, one limitation is the established alteration of circadian rhythm of bone resorption in women with osteoporosis, who demonstrate an approximately $15 \%$ increase bone resorption at night relative to healthy postmenopausal women [10]. In addition, the findings in this study relate to the 150-mg sustained-release tablet formulation administered in the fed state, with standardized, nutritionally matched meals. The results may be different with other doses, formulations and administration times of ONO-5334 and also with varied diets.

In conclusion, morning administration of ONO-5334 150mg SRT for 5 days conferred $>60 \%$ suppression of CTX-I sustained across $24 \mathrm{~h}$, compared with $12 \mathrm{~h}$ after evening administration. Morning administration achieved 6\% greater suppression of serum CTX-I 24-h AUE $(P<.05)$ and 7\% greater suppression of urinary CTX-I/creatinine 24-h AUE $(P<.01)$ compared with evening administration. Despite these differences, evening administration also appeared efficacious in suppressing markers of bone resorption. Future studies will clarify whether morning dosing would show increased efficacy with regard to BMD and whether the morning-versus-evening effect is observed with higher doses of ONO-5334.

Acknowledgments The authors would like to thank the subjects and staff who participated in the study and Helen Lambert and Susan Lanham-New for their valuable work on the nutritional aspects of the study. Isabel Brough, Ph.D., and Laila Guzadhur, Ph.D., of Niche Science $\&$ Technology provided writing and editorial support during the preparation of this manuscript, and this service was contracted by Ono Pharma UK Ltd. The data and analysis were independently checked by the first author (Richard Eastell) with the help of a statistician (Mr. Mike Bradburn), employed by the University of Sheffield.

Funding This study was funded by ONO Pharmaceutical Ltd.

Conflicts of interest Maria Small, John Sharpe, Hiroyuki Yamada, Mikihiro Yuba, Mitsunobu Tanimoto, and Steve Deacon are employees of the study sponsor, Ono Pharmaceutical Co, Ltd (Japan), or its European office, Ono Pharma UK Ltd (UK). Hiroyuki Yamada, Mikihiro Yuba, and Mitsunobu Tanimoto have stock options of Ono Pharmaceutical Co, Ltd. Richard Eastell, Derk-Jan Dijk, and Aldona Greenwood provided the study sponsor with scientific advice and received consultancy fees as members of the study's Steering Committee.

Open Access This article is distributed under the terms of the Creative Commons Attribution-NonCommercial 4.0 International License (http:// creativecommons.org/licenses/by-nc/4.0/), which permits any noncommercial use, distribution, and reproduction in any medium, provided you give appropriate credit to the original author(s) and the source, provide a link to the Creative Commons license, and indicate if changes were made.

\section{References}

1. National Osteoporosis Foundation (2013) Clinician's guide to prevention and treatment of osteoporosis. National Osteoporosis 
Foundation, Washington, DC, Available at: http://nof.org/files/ nof/public/content/resource/913/files/580.pdf. Accessed 21 October 2013

2. Miller PD, Jamal SA, Evenepoel P, Eastell R, Boonen S (2013) Renal safety in patients treated with bisphosphonates for osteoporosis: a review. J Bone Miner Res 28:2049-59

3. Khosla S, Bilezikian JP, Dempster DW et al (2012) Benefits and risks of bisphosphonate therapy for osteoporosis. J Clin Endocrinol Metab 97:2272-82

4. Langdahl B, Binkley N, Bone $\mathrm{H}$ et al (2012) Odanacatib in the treatment of postmenopausal women with low bone mineral density: five years of continued therapy in a phase 2 study. J Bone Miner Res 27:2251-8

5. Ochi Y, Yamada H, Mori $\mathrm{H}$ et al (2014) ONO-5334, a cathepsin $\mathrm{K}$ inhibitor, improves bone strength by preferentially increasing cortical bone mass in ovariectomized rats. J Bone Miner Metab 32(6): $645-52$

6. Engelke K, Nagase S, Fuerst T et al (2014) The effect of the cathepsin $\mathrm{K}$ inhibitor ONO-5334 on trabecular and cortical bone in postmenopausal osteoporosis: the OCEAN Study. J Bone Miner Res 29:629-38

7. Nagase S, Hashimoto Y, Small M et al (2011) Effect of a new sustained-release formulation of the novel cathepsin $\mathrm{K}$ inhibitor, ONO-5334, on serum and urine biochemical markers of bone turnover in healthy post-menopausal women. Poster (SA448/FR448) presented at the ASBMR, San Diego

8. Hasegawa C, Ohno T, Umemura T et al (2014) Population pharmacokinetic and pharmacodynamic modeling of different formulations of ONO-5334, cathepsin K inhibitor, in Caucasian and Japanese postmenopausal females. J Clin Pharmacol 54:23-34

9. Eastell R, Calvo MS, Burritt MF et al (1992) Abnormalities in circadian patterns of bone resorption and renal calcium conservation in type I osteoporosis. J Clin Endocrinol Metab 74:487-94

10. Eastell R, Simmons PS, Colwell A et al (1992) Nyctohemeral changes in bone turnover assessed by serum bone Gla-protein concentration and urinary deoxypyridinoline excretion: effects of growth and ageing. Clin Sci 83:375-82

11. Schlemmer A, Hassager C, Jensen SB, Christiansen C (1992) Marked diurnal variation in urinary excretion of pyridinium crosslinks in premenopausal women. J Clin Endocrinol Metab 74:47680

12. Bjarnason NH, Henriksen EEG, Alexandersen P, Christgau S, Henriksen DB, Christiansen C (2002) Mechanism of circadian variation in bone resorption. Bone 30:307-13

13. Ahmad AM, Hopkins MT, Fraser WD, Ooi CG, Durham BH, Vora JP (2003) Parathyroid hormone secretory pattern, circulating activity, and effect on bone turnover in adult growth hormone deficiency. Bone 32:170-9

14. Blumsohn A, Herrington K, Hannon RA, Shao P, Eyre DR, Eastell $R$ (1994) The effect of calcium supplementation on the circadian rhythm of bone resorption. J Clin Endocrinol Metab 79:730-5

15. Karsdal MA, Byrjalsen I, Riis BJ, Christiansen C (2008) Investigation of the diurnal variation in bone resorption for optimal drug delivery and efficacy in osteoporosis with oral calcitonin. BMC Clin Pharmacol 8:12

16. Karsdal MA, Henriksen K, Bay-Jensen AC et al (2011) Lessons learned from the development of oral calcitonin: the first tablet formulation of a protein in phase III clinical trials. J Clin Pharmacol 51:460-71

17. Buysse DJ, Reynolds CF, Monk TH, Berman SR, Kupfer DJ (1989) The Pittsburgh Sleep Quality Index (PSQI): a new instrument for psychiatric research and practice. Psychiatr Res 28:193-213

18. Akerstedt T, Hume K, Minors D, Waterhouse J (1994) The subjective meaning of good sleep, an intraindividual approach using the Karolinska Sleep Diary. Percept Mot Skills 79(1 Part 1):287-96
19. Christgau S, Bitsch-Jensen O, Hanover Bjarnason N, Gamwell Henriksen E, Qvist P, Alexandersen P, Bang Henriksen D (2000) Serum CrossLaps for monitoring the response in individuals undergoing antiresorptive therapy. Bone 26(5):505-11

20. Vasikaran $S$, Eastell R, Bruyère $O$ et al (2011) Markers of bone turnover for the prediction of fracture risk and monitoring of osteoporosis treatment: a need for international reference standards. Osteoporos Int 22(2):391-420

21. Rogers A, Glover SJ, Eastell R (2009) A randomised, doubleblinded, placebo-controlled, trial to determine the individual response in bone turnover markers to lasofoxifene therapy. Bone 45:1044-52

22. Ng KW (2012) Potential role of odanacatib in the treatment of osteoporosis. Clin Interv Aging 7:235-47

23. Stoch SA, Zajic S, Stone JA et al (2013) Odanacatib, a selective cathepsin $\mathrm{K}$ inhibitor to treat osteoporosis: safety, tolerability, pharmacokinetics and pharmacodynamics - results from single oral dose studies in healthy volunteers. Br J Clin Pharmacol 75:1240-54

24. Stoch SA, Zajic S, Stone J et al (2009) Effect of the cathepsin K inhibitor odanacatib on bone resorption biomarkers in healthy postmenopausal women: two double-blind, randomized, placebocontrolled phase I studies. Clin Pharmacol Ther 86:175-82

25. Bone HG, McClung MR, Roux C et al (2010) Odanacatib, a cathepsin-K inhibitor for osteoporosis: a two-year study in postmenopausal women with low bone density. J Bone Miner Res 25: 937-47

26. Eastell R, Nagase S, Ohyama M et al (2011) Safety and efficacy of the cathepsin K inhibitor ONO-5334 in postmenopausal osteoporosis: the OCEAN Study. J Bone Miner Res 26:1303-12

27. Eastell R, Nagase S, Small M et al (2014) Effect of ONO-5334 on bone mineral density and biochemical markers of bone turnover in postmenopausal osteoporosis: 2-year results from the OCEAN Study. J Bone Miner Res 29:458-66

28. Greenspan SL, Rosen HN, Parker RA (2000) Early changes in serum N-telopeptide and C-telopeptide cross-linked collagen type 1 predict long-term response to alendronate therapy in elderly women. J Clin Endocrinol Metab 85:3537-40

29. Lehmann HJ, Mouritzen U, Christgau S, Cloos PA, Christiansen C (2002) Effect of bisphosphonates on cartilage turnover assessed with a newly developed assay for collagen type II degradation products. Ann Rheum Dis 61:530-33

30. Bettica P, Bevilacqua M, Vago T, Masino M, Cucinotta E, Norbiato G (1997) Short-term variations in bone remodeling biochemical markers: cyclical etidronate and alendronate effects compared. J Clin Endocrinol Metab 82:3034-9

31. Vasikaran S, Eastell R, Bruyère O et al (2011) IOF-IFCC Bone Marker Standards Working Group. Markers of bone turnover for the prediction of fracture risk and monitoring of osteoporosis treatment: a need for international reference standards. Osteoporos Int 22:391-420

32. Reid DM, Hosking D, Kendler D et al (2006) Alendronic acid produces greater effects than risedronic acid on bone density and turnover in postmenopausal women with osteoporosis: results of FACTS International. Clin Drug Invest 26:63-74

33. Black DM, Delmas PD, Eastell R et al (2007) HORIZON Pivotal Fracture Trial. Once-yearly zoledronic acid for treatment of postmenopausal osteoporosis. N Engl J Med 356:1809-22

34. Brown JP, Prince RL, Deal C et al (2009) Comparison of the effect of denosumab and alendronate on BMD and biochemical markers of bone turnover in postmenopausal women with low bone mass: a randomized, blinded, phase 3 trial. J Bone Miner Res 24:153-61

35. Nagase S, Ohyama M, Hashimoto Y, Small M, Kuwayama T, Deacon S (2012) Pharmacodynamic effects on biochemical markers of bone turnover and pharmacokinetics of the cathepsin $\mathrm{K}$ inhibitor, ONO-5334, in an ascending multiple-dose, phase 1 study. J Clin Pharmacol 52:306-18 
36. Baraldo M (2008) The influence of circadian rhythms on the kinetics of drugs in humans. Exp Opin Drug Metab Toxicol 4:175-92

37. Jespersen CM, Frederiksen M, Hansen JF, Klitgaard NA, Sørum C (1989) Circadian variation in the pharmacokinetics of verapamil. Eur J Clin Pharmacol 37:613-5

38. Eisman JA, Bone HG, Hosking DJ et al (2011) Odanacatib in the treatment of postmenopausal women with low bone mineral density: three-year continued therapy and resolution of effect. J Bone Miner Res 26:242-51

39. Miller PD, Bolognese MA, Lewiecki EM et al (2008) Amg Bone Loss Study Group. Effect of denosumab on bone density and turnover in postmenopausal women with low bone mass after long-term continued, discontinued, and restarting of therapy: a randomized blinded phase 2 clinical trial. Bone 43:222-9

40. Rünger TM, Adami S, Benhamou CL et al (2012) Morphea-like skin reactions in patients treated with the cathepsin $\mathrm{K}$ inhibitor balicatib. J Am Acad Dermatol 66:e89-e96

41. Gossiel F, Finigan J, Jacques R et al (2014) Establishing reference intervals for bone turnover markers in healthy postmenopausal women in a nonfasting state. Bonekey Rep 3:573 